\title{
Space Continuity Constraint in Dynamic Flex- Grid/SDM Optical Core Networks: An Evaluation with Spatial and Spectral Super-channels
}

\author{
Rubén Rumipamba-Zambrano ${ }^{(1)}$, Francisco-Javier Moreno-Muro ${ }^{(2)}$, Jordi Perelló( ${ }^{(1)}$, Pablo Pavón- \\ Mariño ${ }^{(2)}$, Salvatore Spadaro ${ }^{(1)}$
}

(1) Advanced Broadband Communications Center (CCABA), Universitat Politècnica de Catalunya (UPC), Jordi Girona 1-3, 08034 Barcelona, Spain, e-mail: rrumipam@ac.upc.edu

(2) Universidad Politécnica de Cartagena (UPCT), Plaza del Hospital 1, 30202 Cartagena, Spain.

\begin{abstract}
Space Division Multiplexing (SDM) appears as a promising solution to overcome the capacity limits of single-mode optical fibers. In Flex-Grid/SDM optical networks, nodes offering full interconnection between input/output fiber ports and spatial channels, typical SDM-Reconfigurable Optical Add/Drop Multiplexer (SDM-ROADM) referred to as independent switching with lane support (InS with LC support), require very complex and expensive node architectures. Alternative designs have been proposed to relax their requirements, such as those realizing Joint-switching (JoS) by switching one spectrum slice across all spatial channels at once. In this work, we evaluate the benefits of a cost-effective SDM-ROADM architecture that makes a trade-off between (i) performance in terms of network throughput and (ii) architectural complexity by forcing the Space Continuity Constraint (SCC) end-to-end, that is, along the connection physical path. The performance and architectural complexity of such a SDM-ROADM solution are compared in dynamic FlexGrid/SDM scenarios against benchmark networks based on InS with LC support and JoS SDM-ROADMs, under both spatial and spectral super-channels. We quantify the network throughput when scaling the spatial multiplicity from 7 to 30 spatial channels, considering Multi-Fiber (MF) as well as Multi-Core Fiber (MCF) SDM solutions. The obtained results reveal that differences in terms of network throughput employing InS without LC support SDM-ROADMs is merely up to $14 \%$ lower than InS with LC support SDM-ROADMs, while the network CAPEX can be dramatically reduced by $86 \%$. In contrast, networks employing InS without LC support SDM-ROADMs carry up to $40 \%$ higher throughput than JoS ones, whereas the network CAPEX can be raised up to $3 x$. This paper also analyses the spatial multiplicity impact on both network metrics (throughput and CAPEX).
\end{abstract}

\section{List of acronyms and nomenclature}

$\begin{array}{ll}\text { AWG } & \text { Arrayed Waveguide Grating } \\ \text { AoD } & \text { Architecture on demand } \\ \text { BBP } & \text { Bandwidth Blocking Probability } \\ \text { B\&S } & \text { Broadcast-and-select } \\ \text { BV-OXC } & \text { Bandwidth Variable Optical Cross Connect }\end{array}$

(C) 2018 Elsevier. This manuscript version is made available under the CC-BY-NC-ND 4.0 license http://creativecommons.org/licenses/by-nc-nd/4.0/ 


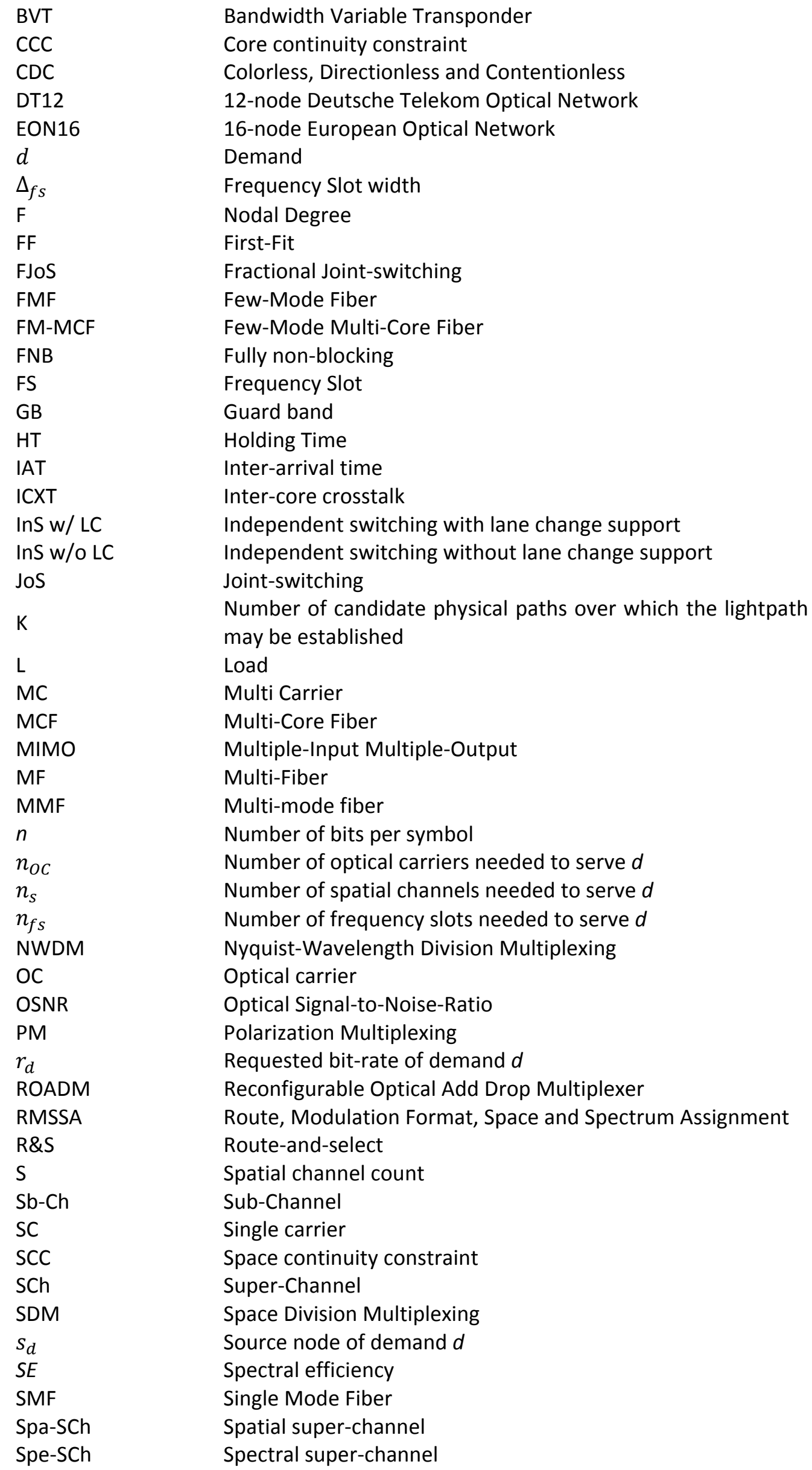




$\begin{array}{ll}\text { S2-SCh } & \text { Spectral-spatial super-channel } \\ \text { SP } & \text { Shortest path } \\ \text { SSS } & \text { Spectrum Selective Switch } \\ t_{d} & \text { Destination node of demand } d \\ \text { TP } & \text { Traffic profile } \\ \text { TR } & \text { Transmission reach } \\ \text { WDM } & \text { Wavelength Division Multiplexing }\end{array}$

\section{Introduction}

Flex-Grid [1] and Space Division Multiplexing (SDM) [2] technologies have captured large interest among the research community in the recent years, on the way to find out novel solutions that optimize the spectrum usage and overcome the capacity limit of the single mode fibers (SMFs), bounded by the so-called non-linear Shannon's limit.

Flex-Grid discretizes the optical spectrum in frequency slots (FSs) of $12.5 \mathrm{GHz}$ width, as recommended by the ITU-T in [3]. Unlike Wavelength Division Multiplexing (WDM) networks, Flex-Grid allows transmissions at flexible bit-rates, relying on the programmability of several key parameters, such as the modulation format, symbol rate, sub-channel multiplicity, among others. Ultra-high bit-rate transmissions are possible in Flex-Grid by concatenating multiple adjacent Sub-Channels (Sb-Chs), thus forming a Super-Channel (SCh). These programmable functions are supported by several network elements, like the Bandwidth Variable Transponders (BVTs) that transmit/receive optical signals based on Single Carrier (SC) or MultiCarrier (MC) systems. The transmitted optical signals are subsequently switched and filtered (commonly referred to as bypass operation) at intermediate Bandwidth Variable Optical Cross Connect (BV-OXC) nodes. In particular, BV-OXCs with additional add/drop capabilities for connections that originate/terminate at the specific network node are implemented as Reconfigurable Optical Add/Drop Multiplexers (ROADMs), based on Spectrum Selective Switches (SSSs).

In its turn, SDM is considered as the "next-frontier" of fiber optics, able to scale up the capacity of current WDM optical fiber systems [4]. Several SDM flavors capable of multiplying the number of available spatial channels are currently under research. As a starting point, SMFs of current telecom operators can be upgraded to bundles of parallel SMFs, acting as a Multi-Fiber (MF) solution. However, novel fiber designs [5] are also attractive to facilitate SDM system integration. For example, the spatial multiplicity can rely on the transmission of different fiber modes; such is the case of the Multi-Mode Fibers (MMFs) or Few-Mode Fibers (FMFs). Another alternative solution is to increase the number of cores within each fiber cladding, typically referred to as Multi-Core Fibers (MCFs). Moreover, if these cores can carry few modes each, this kind of fibers are called Few-Mode Multi-Core Fibers (FM-MCFs). The coupling between modes or cores is a new impairment to be considered in SDM, in order to determine if equalization, typically based on Multiple-Input and Multiple-Output (MIMO) processing, is necessary. MCFs have become particularly attractive given their extremely low inter-core crosstalk (ICXT) values [6]-[10], avoiding the need of MIMO equalization, even over long-haul transmissions. This type of fibers are referred to as weakly-coupled MCFs, for which we estimated in our previous work [11] the transmission reach (TR) as the most restrictive limitation, either imposed by Optical Signal-to-Noise-Ratio (OSNR) or worst aggregate ICXT. 
Several SDM-capable ROADM (SDM-ROADM) designs have been proposed in the literature aimed at relaxing the hardware requirements and dealing with XT [12][13]. The most flexible SDM-ROADM architecture, but also the most expensive solution, provides full interconnection between ports and spatial channels (fibers/cores/modes). Thus, any spectral portion from any spatial channel in any input port can be directed independently to any spatial channel in any output port. In addition, if Colorless, Directionless and Contentionless (CDC) property is enabled at such nodes, then full flexibility with no internal blocking is provided. This SDMROADM has been called "space-wavelength switching granularity with SDM lane change" in [12], [14], while in [11], [15] is referred to as fully non-blocking (FNB) and in [16], [17] as independent switching (InS) with lane change (LC) support. As seen, different names have been used in the literature for the same SDM-ROADM. Aiming at unifying nomenclature, throughout this paper we adopt the acronym InS with LC support when we refer to it. Initial works in the literature present allocation schemes for MCF networks assuming InS with LC support SDM-ROADMs (e.g., see [18], [19]). However, the complexity and cost of such SDMROADM architectures is enormous. Indeed, when conventional ROADMs for SMFs turn into SDM-ROADMs and each input/output fiber must support $S$ spatial channels: (i) the number of required SSS devices at ROADMs must be multiplied by $S$, and (ii) its individual size is also multiplied by $S$, resulting in an $S^{2}$ complexity increase.

To relax the complexity of InS with LC support, the so-called Architecture on Demand (AoD) [13] avoid underutilized hardware modules by sharing them on demand via node programmability capabilities. All input/output ports, add/drop ports, as well as hardware components, like SSSs, splitters/combiners, amplifiers, mux/demux, etc., are connected to a backplane and interconnected in a customized manner according to the network traffic requirements. However, processing complexity and resilience mechanisms of the backplane are important aspects to be considered. Reference [20] assumes this architecture to allocate spectral super-channels (Spe-SChs).

Another more restrictive, but less complex switching strategy, is the so-called Joint-switching (JoS) [21], which jointly switches one spectrum slice across all spatial channels at once. All spatial channels are treated as a single entity and this technique is required for stronglycoupled SDM fibers. An intermediate solution between InS with LC support and JoS, called Fractional Joint-Switching (FJoS) [12] is also possible where the spatial channels are grouped into subgroups and JoS is independently applied to any of them. In FJoS, the smaller the subgroup size, the higher the flexibility in the spatial domain. For the interested reader, recent work [14] includes an analysis of the node complexity and cost of different SDM-ROADM architectures.

In our previous works [11], [15] we assessed in offline (i.e., static) network scenarios one additional SDM-ROADM architecture that trades spatial channel switching for superior costefficiency. This architecture introduces the Space (generalization of Core) Continuity Constraint (SCC), i.e., it forces to employ the same spatial channel index along the end-to-end routing path. This SDM-ROADM is called "space-wavelength switching granularity without SDM lane change" in [14], [22], while in other works, similar to what happens with InS w/ LC support, it has been called using different names, e.g. only "InS" in [23], [24], "Ind-Sw" in [25]-[27] or CCC-ROADM in [11], [15]. Again, aiming at unifying terminology, we adopt the acronym InS without LC support when we refer to it. Particularly, the obtained results in [11], [15] revealed 
minor network throughput reductions (up to $10 \%$ at most) in exchange of very important complexity reductions deploying this SDM-ROADM versus the InS with LC support one.

As a follow-up to [11], [15], in this work we consider two benchmark SDM-ROADM architectures, namely, Ind-Sw with LC support and JoS previously described, to evaluate for the first time to the best of our knowledge, the benefits of SCC implemented by means of InS without LC support SDM-ROADM in online (i.e., dynamic) network scenarios. This is a crucial needed validation, since in dynamic scenarios spectrum fragmentation is an intrinsic issue [22] affecting the overall network performance of Flex-Grid/SDM networks, even if fragmentationaware heuristics are considered [28], [29]. Previous works evaluate the performance of different architectures (InS without LC support, JoS and FJoS) under static [17], [25] and dynamic [24], [26] scenarios. Reference [26] states that the performance (in terms of blocking probability) of different SDM-ROADMs are influenced by the traffic profile. In addition to this, we aim to analyze the impact of the spatial multiplicity. For this purpose, we scale the spatial channel count from 7 to 30 (first SDM region to be explored [30]), considering not only crosstalk-free SDM fibers (like in all previous works [17], [24]-[26]), but also SDM fibers with the presence of ICXT, corresponding to MCF laboratory prototypes available in the literature [6]-[10]. We also account for Sb-Chs arranged over both the spectral domain -i.e., spectral super-channel (Spe-SCh) and over spatial domain -i.e., spatial super-channel (Spa-SCh)-. Finally, we contrast the performance (in terms of throughput) of different SDM-ROADMs versus their hardware requirements, especially at the bypass part.

The remainder of this paper is structured as follows. Section 2 describes the different SDMROADM architectures considered for Flex-Grid/SDM network scenarios. Section 3 elaborates on the SCh allocation supported by different SDM-ROADM architectures. For this, subsection 3.1 presents a heuristic mechanism for resource allocation considering SCC. Next, section 4 presents numerical results and subsequent discussion in two subsections. Subsection 4.1 presents the scenario details and assumptions, while subsection 4.2 describes the analysis of the network throughput scalability, together with the cost analysis. Finally, section 5 draws up the main conclusions and envisions future research lines.

\section{SDM-ROADM Architectures}

ROADMs are in charge of automatically adding, dropping or bypassing lightpaths in Flex-Grid Optical Networks. The ROADM design includes the SSS devices, which are able to switch any frequency slot (FS) at any of its input ports, to any of its outputs ports [31]. The SSSs are the most expensive elements in the ROADM design.

ROADM functions are typically implemented based on two alternative schemes, the so-called Broadcast-and-Select (B\&S) and the Route-and-Select (R\&S) one. On the one hand, the B\&S scheme offers cost, power consumption and optical/electronic complexity reductions, as well as low overall system penalties. On the other hand, the R\&S practically doubles the number of SSSs to provide superior isolation on the blocking ports and a low insertion loss regardless of the port count [32]. According to [32], the B\&S architecture seems the best choice for ROADMs with nodal degree $(F)$ lower or equal than 9 , while for $F>9$, R\&S benefits compensate its extra cost. Commercial ROADMs commonly use the B\&S scheme to switch traffic. However, for next generation optical networks deploying high-port-count Spectrum Selective Switches (SSSs) that will require high isolation between ports, the prevalence of the R\&S scheme is foreseen [33]. 
The full interconnection between input ports (and spatial channel index) and output ports (and spatial channel index) in an InS with LC support SDM-ROADM [Fig. 1(a)] provides a fully flexible spectral and spatial channel configuration. This design has the finest switching granularity, allowing any wavelength (therefore any SCh configuration) to be switched among all available spatial channels. In contrast, it requires a huge amount of SSSs, two per spatial channel and degree. The design of Fig. 1(a) also includes one CDC-capable (by means of a multicast switch [33]) Add/Drop module to inject/extract channels originated/terminated at the node without any internal blocking.

Such an InS with LC support SDM-ROADM design entails significant complexity and cost. To address this issue, some alternative designs have been proposed:

1) The JoS strategy allows to switch one spectrum slice in all spatial channels at once (i.e., spatial super-channels are mandatory to be configured). This latter aspect allows reducing the number of SSSs to only two per degree. In contrast, the number of required ports per SSS is multiplied by $S$ (the total number of spatial channels per fiber link). That is, for a JoS SDM-ROADM [Fig. 1(b)], the conventional SSS of $1 \times F$ ports has to scale up to $S \times(1 \times F)$ ports. This new configuration is supported by reprogramming conventional SSSs. For example, a $7 \times(1 \times 2)$ SSS can be configured by means of $1 \times 20$ conventional SSSs [21]. Under this design, the number of required SSSs per node is $S$ times lower than those required by InS with LC support SDM-ROADMs.

2) Another alternative to simplify node design is to introduce a constraint in the channel routing, namely, the previously introduced SCC. This design consideration has the advantage of reducing the internal SDM-ROADM connections between all ports (and spatial channels) forcing to use a unique spatial channel index $\left(s_{i}\right)$ along the end-to-end lightpaths. Such InS without LC support SDM-ROADM is depicted in Fig. 1(c), where spatial channels are separated (e.g., the cores by means of MCF breakout [12], [21]) and each $s_{i}$ of each fiber port is connected to a conventional ROADM (as the ones used for SMFs). In fact, we have one ROADM per $s_{i}$ and all $s_{i}$ belonging to all input/output fiber ports are multiplexed/demultiplexed in ROADM $i$. As the InS without LC support SDM-ROADM can be built as a parallel arrangement of $S$ conventional ROADMs, each SSS has $1 \times F$ ports and the total number of SSSs per SDM-ROADM is $2 \cdot S \cdot F$ taking into account the R\&S scheme as well. It is worth mentioning that, InS without LC support SDM-ROADM can be built also following the B\&S scheme by only deploying passive splitters at input ports instead of SSSs. In fact, in these SDM-ROADMs, the port count of the SSSs only depends on the nodal degree $F$. Hence, having $F<9$ (i.e., not a high isolation is demanded), the $B \& S$ scheme would be perfectly feasible [22], thus saving further costs. Furthermore, InS without LC support SDM-ROADM also supports any SCh configuration. 
(a)

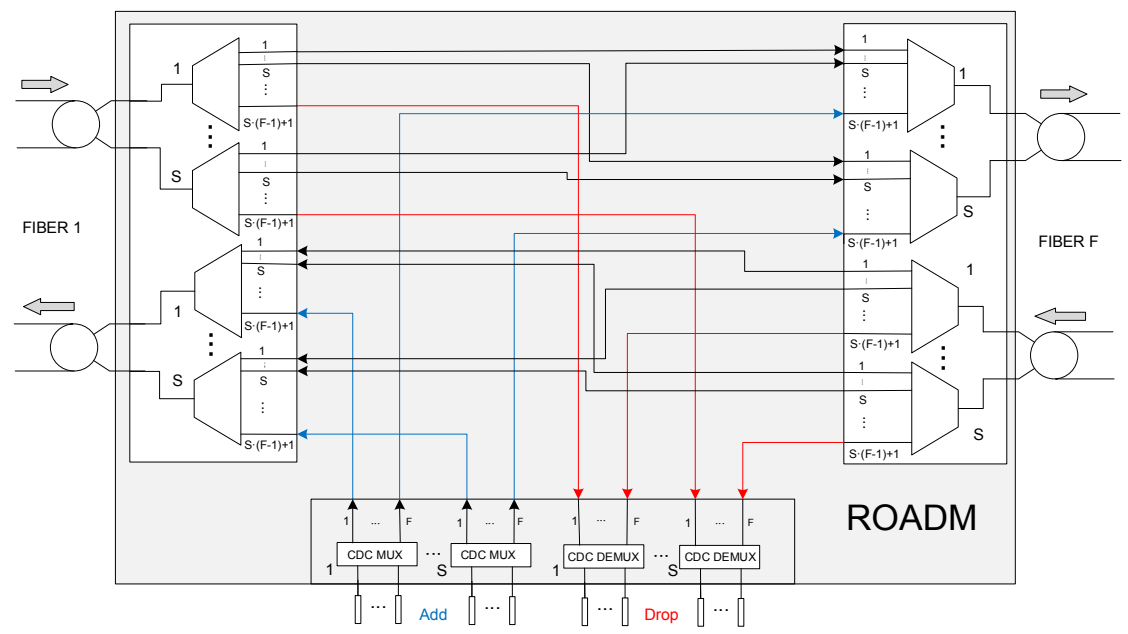

(b)

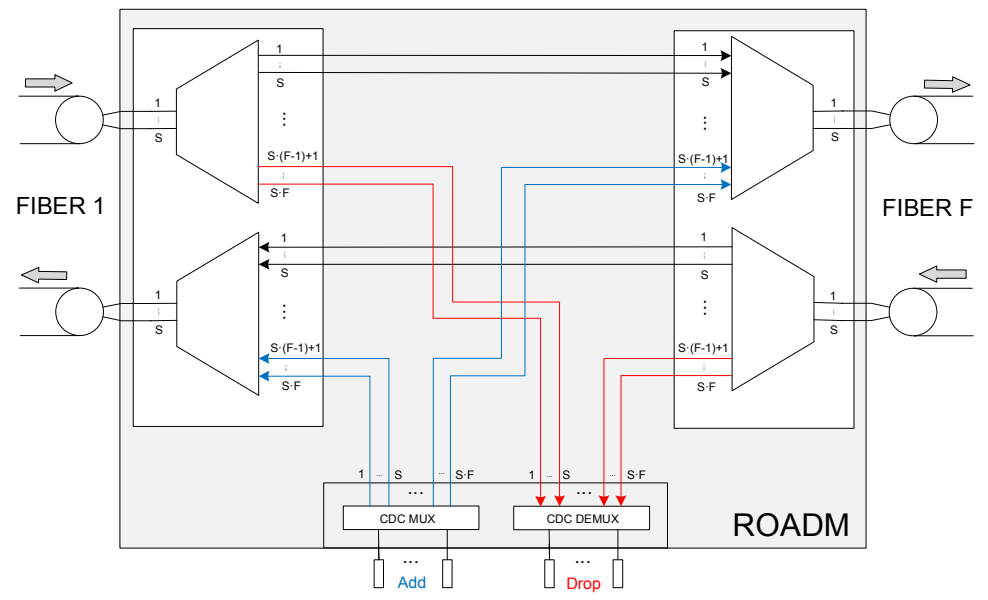

(c)

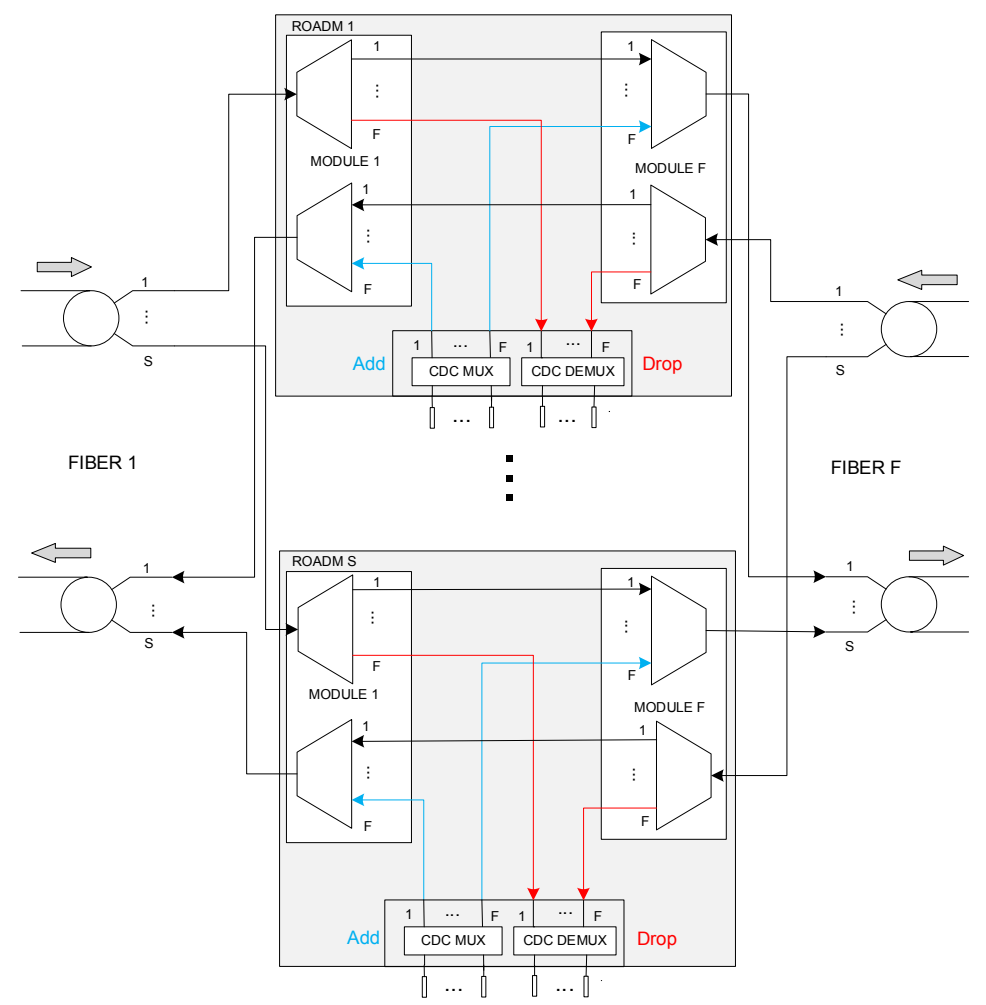

Fig. 1. SDM-ROADM architectures: (a) InS w/ LC support, (b) JoS, and (c) InS w/o LC support, only two degrees are shown for simplicity. 
Table 1 summarizes the quantification of the number of SSSs per degree and its size from previous Fig. 1 and also presented in [16], [17]. Reference [16] provides general information about SSS parameters for group of spatial channels switched with or without LC support. As observed, the required number of SSSs by InS ( $\mathrm{w} /$ or w/o LC support) is $S$ times more than the required by the JoS one. Conversely, the port count per SSS (therefore, SSS size) in InS ( $w /$ and w/o LC support) SDM-ROADMs is lower than in JoS ones. That is, JoS SDM-ROAMDs demand a lower number of SSSs in exchange for increasing their size. It is worth noticing that currently available commercial SSSs (with maximum size of $1 \times 32$ [34]) could not support JoS. Instead, high-port-count SSSs are necessary, for example, for $S=7$ and $F=4$, at least 35 ports would be required. However, although these high-port-count SSSs are not commercially available yet, it is foreseen that cost per port decreases with the SSS size [16] (e.g. see table 4 presented later on in subsection 4.2).

TABLE 1

SSSs metrics for different SDM-ROADM designs

\begin{tabular}{|c|c|c|c|c|}
\hline SDM-ROADM & $\begin{array}{c}\text { Number of SSSs } \\
\text { per Degree }\end{array}$ & SSS port count & SSS size & $\begin{array}{c}\text { Supported } \\
\text { SCh types }\end{array}$ \\
\hline InS w/o LC support & $\mathbf{2} \cdot \boldsymbol{S}$ & $\boldsymbol{F}+\mathbf{1}$ & $\mathbf{1} \times \boldsymbol{F}$ & All \\
\hline InS w/ LC support & $2 \cdot S$ & $S \cdot(F-1)+2$ & $1 \times[S \cdot(F-1)+1]$ & All \\
\hline JoS & 2 & $S+S \cdot F$ & $S \times(1 \times F)$ & Spa-SCh \\
\hline
\end{tabular}

\section{Super-channel allocation supported by SDM-ROADMs}

In this section, we briefly present the main characteristics of different SCh configurations supported by SDM-ROADMs. The introduction of the space dimension enables different SCh allocation options, as described in [22]. Indeed, the SCh configuration depends on how its SbChs are arranged across both spectral and spatial domains. For instance, Sb-Chs of a spectral super-channel (Spe-SCh) are contiguously allocated in the spectral domain over the same spatial channel using MC systems, whereas those of a spatial super-channel (Spa-SCh) are allocated across the spatial domain over a SC. The accommodation of multiple Sb-Chs across both spatial and spectral domains, namely, spectral-spatial super-channel ( $\mathrm{S2}-\mathrm{SCh}$ ) is also feasible in SDM networks. Results reported in [23] show that Spe-SChs may yield better performance (in terms of Bandwidth Blocking Probability, BBP), as they require much less spectral overhead due to Guard Bands (GBs). In fact, GBs between Sb-Chs can be eliminated by employing Nyquist-Wavelength Division Multiplexing (NWDM) [35], whilst GBs between SChs are always necessary.

Fig. 2 illustrates different SCh configurations. Spa-SCh shown in Fig. 2(a) requires a single Optical Carrier (OC) at frequency $f_{1}$, whilst the S2-SCh of Fig. 2(b) is configured with two OCs at frequencies $f_{1}$ and $f_{2}$. S2-SCh can be seen as a MC Spa-SCh. According to the definition of JoS operation, it requires that the traffic demands be allocated in the form of (SC or MC) Spa-SChs. In fact, the allocation of the same spectrum portion in all $(S)$ spatial channels allows jointly switching all optical channels at once. For this purpose, GBs per spatial Sb-Ch are required to allow SSS filters to properly capture these optical channels. The GB width greatly influences the network performance, because the effective spectral capacity to allocate optical signals is reduced as the GB width gets coarser. That is, depending on the network diameter (longest shortest path between any pair of nodes), network performance can increase by $\sim 80-160 \%$ 
reducing GBs from $12.5 \mathrm{GHz}$ to $0 \mathrm{GHz}$ (ideal case) [36]. From the channel routing point of view, JoS SDM-ROADM treats all spatial channels as if they were a single one. Hence, in order to maximize the network throughput, like in Flex-Grid (over SMFs), the goal is to allocate the narrowest spectral slice per spatial Sb-Ch. Consequently, when a lightpath is established, a spectrum slice is reserved in the $S$ spatial channels over the end-to-end routing path. If the considered lightpath has free spatial channels, they cannot be used by other traffic demands at intermediate nodes, unless modifications in the SDM-ROADMs are introduced [24]. One simple and direct approach is to reuse lighpaths for allocating traffic demands sharing the same source and destination nodes. This can be seen as end-to-end spatial traffic grooming allowing the traffic aggregation only in the source and destination nodes (e.g., see heuristic in [37]).

Fig. 2(c) illustrates a Spe-SCh. As observed, it requires to setup four OCs at frequencies from $f_{1}$ to $f_{4}$, instead of the one or two OCs required for SC or MC Spa-SCh, respectively. If NyquistWDM is employed, GBs between Sb-Chs can be eliminated, requiring only GB/2 at each spectral end of the SCh. That is why Spe-SChs can achieve the best performance. In contrast, a BVT supporting Spa-SChs does not require frequency combs or Arrayed Waveguide Gratings (AWGs) [38], uses shared lasers and joint digital signal processing of spatial sub-channels at the receivers by means of integrated chips [39]. All these aspects potentially reduce the power consumption and cost of the SDM networks.

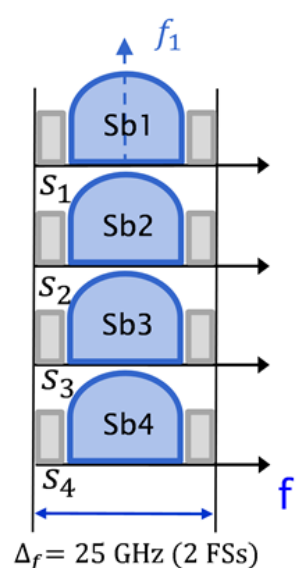

(a)

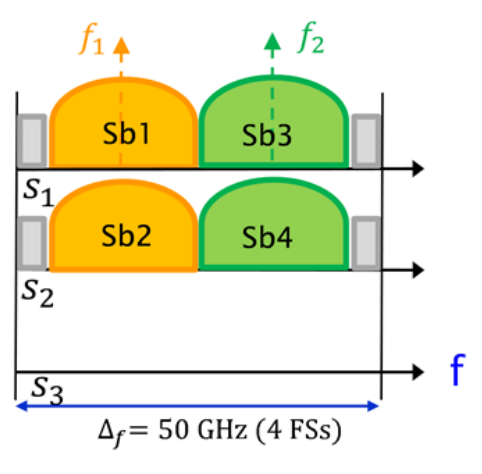

(b)

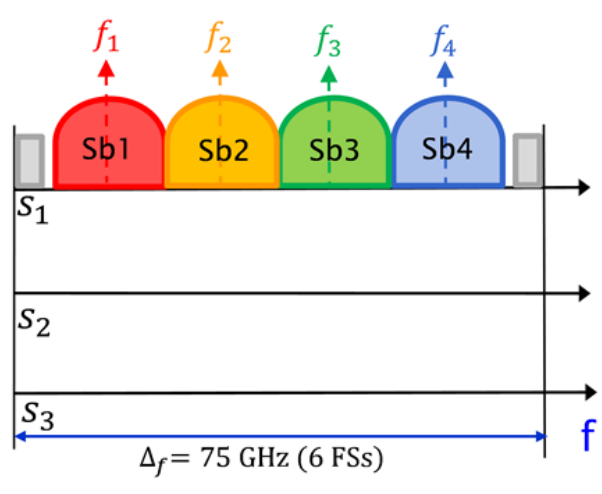

(c)

Fig. 2. SCh allocation schemes: (a) Spa-SCh, (b) S2-SCh and (c) Spe-SCh

Regarding the number of OCs needed to configure the SChs, it depends mainly on the baud rate of the transceivers. In fact, for a given requested bit-rate $r_{d}$, number of spatial channels $n_{S}$ and spectral efficiency (SE) of the modulation format (with $n$ bits/symbol) the number of OCs can be computed by $\left[\frac{r_{d} /\left(n_{s} \cdot S E\right)}{\max (\text { baud_rate })}\right]$. That is, a SCh transmits an aggregate bit-rate equal to $r_{d}=$ baud_rate $\cdot n \cdot n_{O C}$.

InS ( $w /$ and w/o LC support) SDM-ROADMs introduced in Section 2 do not impose any SCh configuration. In fact, they support any of the three SCh types at the same cost (only enforcing the SCC in the case of InS without LC support SDM-ROADM). The spatial and spectral resources can be assigned freely to any traffic demand. As seen in Table 1, Spa-SChs can be routed by all SDM-ROADM designs, while Spe-SChs are only supported by InS (w/ and w/o LC support) SDMROADMs. 


\subsection{Route, Modulation Format, Space and Spectrum Assignment (RMSSA) heuristic}

In this subsection, we present a greedy heuristic for solving the RMSSA problem associated to the allocation of an incoming Spe-SCh/Spa-SCh request in a Flex-Grid/SDM optical network, considering the previously introduced InS without LC support SDM-ROADM architecture. The details of the heuristic are depicted in Pseudo-code 1.

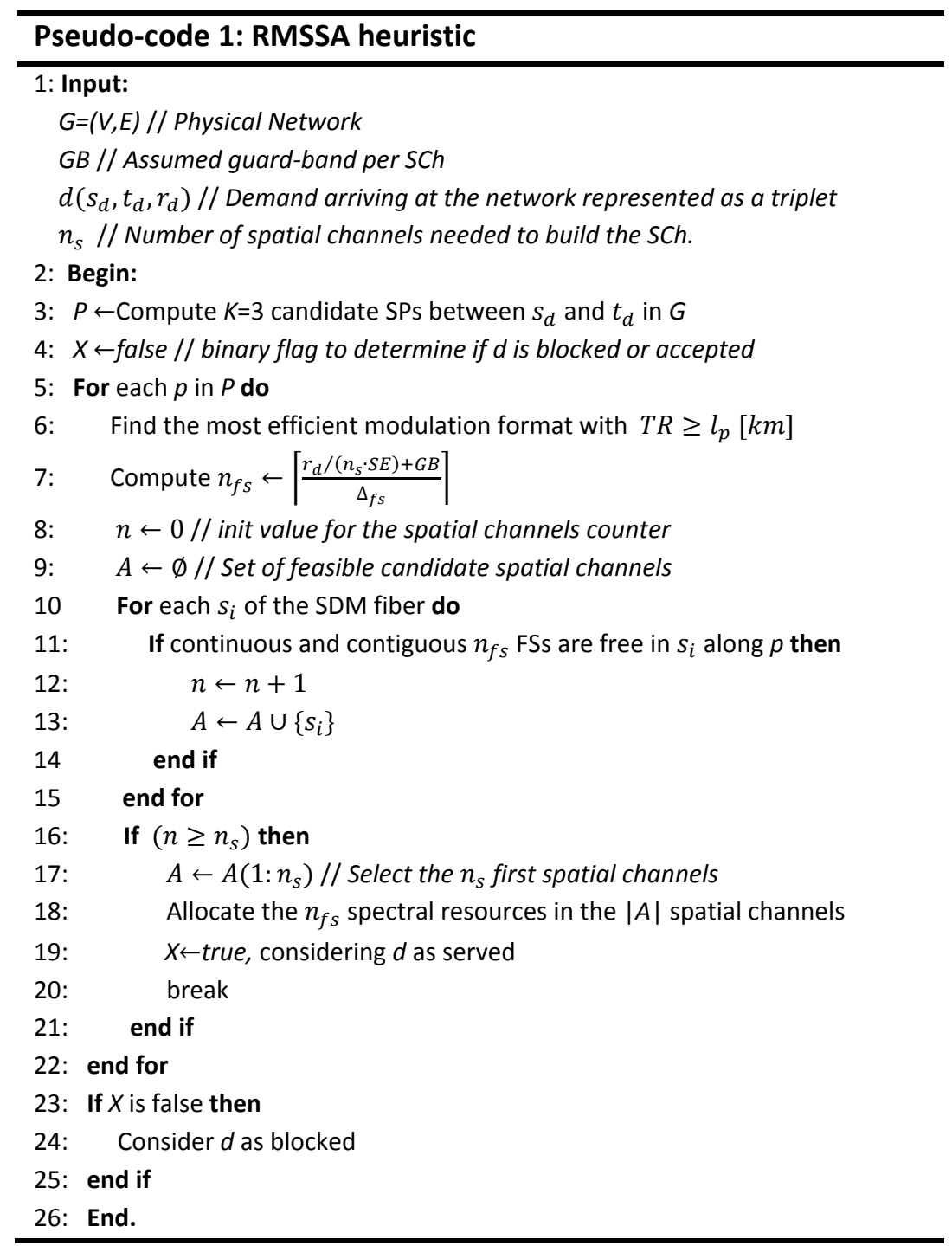

For a given incoming demand $d$, the $K=3$ candidate Shortest Paths (SPs) are computed between its source $\left(s_{d}\right)$ and destination $\left(t_{d}\right)$ nodes, taking their physical length $\left(l_{p}\right)$ as the metric. For each one of them, the most efficient modulation format meeting the transmission reach (TR) requirements is selected. Following the procedure, the number of necessary FSs $\left(n_{f S}\right)$ is calculated as $\left\lceil\frac{r_{d} /\left(n_{s} \cdot S E\right)+G B}{\Delta_{f s}}\right\rceil$, where $r_{d}$ is the bit-rate required by the demand $d, n_{s}$ is the number of spatial channels needed to be assigned, $S E$ is the spectral efficiency of the selected modulation format (in $\mathrm{b} / \mathrm{s} \cdot \mathrm{Hz}$ ) and $\Delta_{f s}$ is the FS width (i.e., $12.5 \mathrm{GHz}$ according to G.694.1 ITU-T recommendation [3]). Obviously, $n_{s}=1$ in the case of a Spe-SCh, while for SpaSCh we compute it based on the heuristic in [37], which essentially seeks for the minimum $n_{S}$ 
value ensuring that the minimum number of FSs are used to allocate it (i.e., the same number of FSs as if all spatial channels would have been assigned). Next, for each spatial channel index $\left(s_{i}\right)$ of the SDM fiber, the candidate path is tested for the allocation of the computed $n_{f s}$ FSs in $s_{i}$ along the routing path, fulfilling both the spectrum continuity and contiguity constraints of the Flex-Grid networks. If the path has enough spectral resources in at least $n_{s}$ spatial channels, the demand is considered served. Otherwise, the next candidate path is explored until no more candidates are available (in such a case, the demand is considered blocked). Note that a First-Fit (FF) strategy is used in the route, space and spectrum assignment, similar to approaches described in [23], [37]. Our goal is to evaluate different SDM-ROADMs with a basic and lightweight resource allocation scheme, although other more advanced schemes (out of the scope of this work), especially for spatial resource allocation can be explored, e.g., least congested routing path in order to balance the network load among the vast amount of spectral-spatial resources.

It is worth highlighting that, the RMSSA heuristic has been carefully designed providing, in average, execution times lower than $1 \mathrm{~ms}$ per demand in a large continental backbone network (16 nodes and 23 links, see detailed description of this network in the next Subsection 4.1) with 30 spatial channels. That is, a lightweight heuristic has been designed, meaning that although the additional constraint introduced (SCC) for Flex-Grid/SDM networks, this does not suppose a substantial computational complexity increase.

\section{Numerical Results}

\subsection{Scenario Details and Assumptions}

In order to evaluate the network throughput deploying different SDM-ROADM designs introduced in Section 2, we have carried out several simulations considering a dynamic network scenario, where demands arrive to the network following a Poisson distribution with given mean inter-arrival and holding times (denoted as IAT and HT, respectively). The traffic is distributed uniformly between all pairs of source-destination nodes. Upon arrival, demands request for a bidirectional lightpath, with a bit-rate selected among two different traffic profiles: TP1 $=\{100,400,1000\} \mathrm{Gb} / \mathrm{s}$ and TP2 $=\{400,1000,2000\} \mathrm{Gb} / \mathrm{s}$, with the same probabilities of $\{0.4,0.3,0.3\}$. Note that the average requested bit-rate per demand under TP1 is $460 \mathrm{~Gb} / \mathrm{s}$, while under TP2 it raises to 1.06 Tbps. That is, TP2 represents a $2.3 x$ increase in size of the connections regarding TP1.

To obtain the numerical results, we have considered the continental 16-node European Optical Network (EON16) topology and the national 12-node Deutsche Telekom Optical Network (DT12), whose main characteristics are shown in Fig. 3. At each network link, we scale the spatial multiplicity $(S)$ from 7 to 30 spatial channels. For MF-based solutions 1-spatial channel granularity is considered, while for MCF-based ones the five best single-mode MCF prototypes found in the literature [6]-[10] are taken into account, i.e., $S \in\{7,12,19,22,30\}$. Each fiber/core is assumed to have 320 available FSs with $\Delta_{f s}=12.5 \mathrm{GHz}$, while the GB width value has been set to $7.5 \mathrm{GHz}$ [36]. Different offered loads (L=HT/IAT) are simulated by fixing the IAT and incrementing the $\mathrm{HT}$ until we obtain a $B B P$ close to $1 \%$ for each spatial multiplicity value. Therefore, the average network throughput (Avg. Carried Traffic) can be computed by averaging all instantaneous carried traffic after each connection is established along the entire simulation. To get statistically relevant results, we offer $5 \times 10^{5}$ demand requests per execution. 
Note that, there exists an initial transient period due to the Poisson process of arrivals before getting the steady state of $1 \%$ target BBP. To isolate this transient period from the statistic, we must either (i) use a relevant time of observation compared with the transient (i.e., use a statistical sample with significant size) or (ii) identify all connections occurred during the transient and directly discard them from the statistic. We use (i), but first we have verified with (ii) that the Avg. Carried Traffic metric remains unaffected.

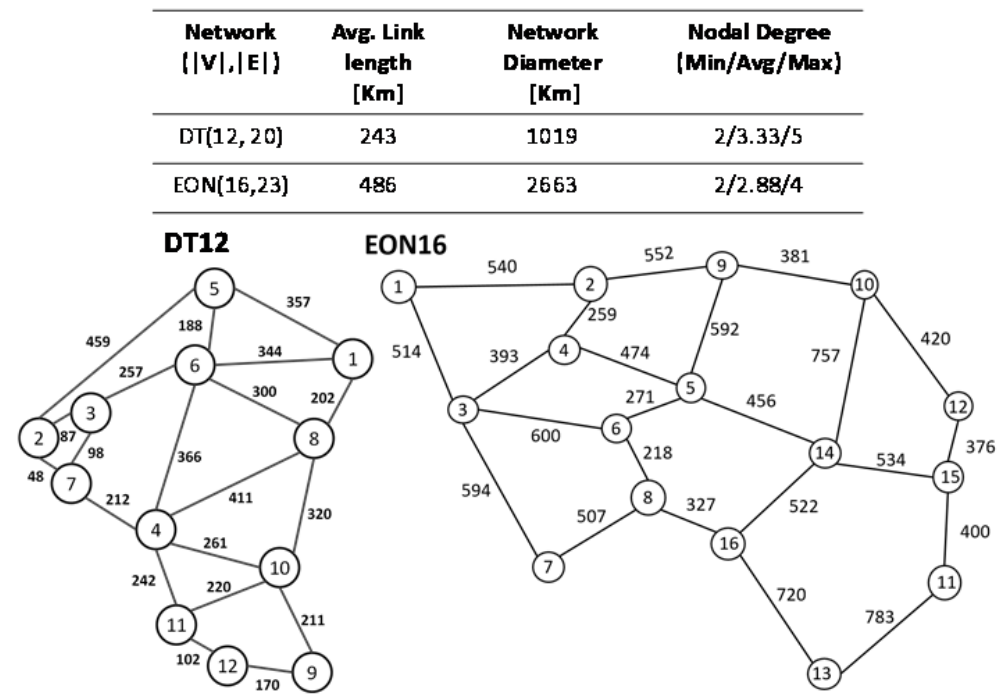

Fig. 3. Network reference topologies with their main characteristics

TR value could be perceived as the minimum value between the one limited by OSNR and the one limited by the worst aggregate ICXT, as presented in [11]. The worst aggregate ICXT values for the considered single-mode MCF prototypes found in the literature are shown in Table 2. The ICXT values correspond to specific fiber layouts. That is why higher core counts can experiment lower ICXT (e.g., 22-core MCF vs. 19-core MCF). All MCFs are homogenous except the 30-core heterogeneous MCF. Moreover, Table 3 shows the overall TR for the same MCFs shown in Table 2. Please note in Table 3 that values in red are the TR limited by the ICXT.

TABLE 2

Measured worst aggregate ICXT at 1550nm

\begin{tabular}{c|c|c|c|c}
\hline \hline $\mathbf{7}$ cores [6] & $\mathbf{1 2}$ cores [7] & $\mathbf{1 9}$ cores [8] & $\mathbf{2 2}$ cores [9] & $\mathbf{3 0}$ cores [10] \\
\hline$-84.7 \mathrm{~dB} / \mathrm{Km}$ & $-61.9 \mathrm{~dB} / \mathrm{Km}$ & $-54.8 \mathrm{~dB} / \mathrm{Km}$ & $-56.2 \mathrm{~dB} / \mathrm{Km}$ & $-60 \mathrm{~dB} / \mathrm{Km}$ \\
\hline
\end{tabular}

TABLE 3

Overall TR estimation in $\mathrm{km}$.

\begin{tabular}{ccccc}
\hline \hline MCF & BPSK & QPSK & 16-QAM & 64-QAM \\
\hline \hline 7 & $>20000$ & 9000 & 2000 & 600 \\
\hline 12 & $>20000$ & 9000 & 2000 & 600 \\
\hline 19 & 4755 & 2383 & 599 & 150 \\
\hline 22 & 6607 & 3311 & 832 & 209 \\
\hline 30 & 15849 & 7943 & 1995 & 501 \\
\hline
\end{tabular}

Finally, we consider that BVTs can operate with three degrees of flexibility, namely, modulation format, baud-rate (max. $32 \mathrm{GBaud}$ ) and Sb-Ch multiplicity, thus obtaining different operational line-rates. 


\subsection{Network Throughput Scalability and Cost Analysis}

In this subsection, we quantify the network throughput deploying InS without LC support SDMROADMs as the one presented in Section 2, aiming at comparing its performance against benchmark scenarios (InS with LC support and JoS SDM-ROADMs), considering both Spa-SChs and Spe-SChs. This analysis is carried out for different spatial multiplicities (scaling $S$ from 7 to 30 spatial channels) including MF and the cases where there exists a MCF prototype. Fig. 4 and Fig. 5 show the average network throughput for different $S$ values for Spe-SCh and Spa-SCh, respectively. We consider the two reference topologies and the two traffic profiles (TP1 and TP2) described in the previous subsection.

Fig. 4 shows the network throughput scalability of the InS without LC support SDM-ROADM vs. InS with LC support one when supporting Spe-SChs. The results show that there are not major differences ( $<2 \%$ ) between both compared architectures (in terms of throughput) in a national network such as the DT12. Conversely, in a continental network as the EON16, quite higher differences, up to $14 \%$, are observed. The fact is that demands in larger networks may require more spectral resources (i.e., less efficient modulation formats are mandatory), hindering the SCh allocation since meeting the SCC gets more complicated. In the EON16 network, it is more clearly evidenced the impact of the spatial multiplicity, namely, the higher the spatial multiplicity $S$, the higher the differences between both SDM-ROADMs performance in terms of throughput (i.e., curves are divergent), which was expectable due to the intrinsic SCC limitation in space channel assignment. Furthermore, in the DT12 network it is also evidenced the TP sensitivity in the network throughput, carrying up to $14 \%$ more traffic for a $2.3 \mathrm{x}$ (TP2/TP1) traffic profile increment (in terms of its average bit-rate per connection). Meanwhile, the TP sensitivity is almost not evidenced for EON16 network.

In particular, 7- , 12-MCFs achieve the same performance as the equivalent MF solutions, since their extremely low ICXT does not become the TR limiting factor, being the OSNR instead as in the equivalent MF solutions. In its turn, 30-core MCF yields very close performance between MCF- and MF-based solutions because although the ICXT is the TR limiting factor, this is not so much different than the one imposed by the OSNR. However, 19- and 22-core MCFs perform noticeably worse than the equivalent MF solutions due to higher ICXT introduced. Between these two MCFs, the performance degradation of 19-core MCF is the most significant because it presents the highest ICXT level, resulting in a network throughput very close to that with 12core MCF. Indeed, the differences between the 19-core MCF and the equivalent MF solution in terms of network throughput are up to $70 \%$ for DT12 and up to $50 \%$ for EON16 network (in the worst cases). The influence of network diameter in the ICXT impact can be explained as follows. In larger networks, path lengths impose less efficient modulation formats by themselves. In addition, as the order of the modulation formats decreases, the TR margin between them increases. For example, according to Table 2 with a 22-core MCF, the TR margin between the 64-QAM and 16-QAM modulation format is $623 \mathrm{~km}(832-209 \mathrm{~km})$, while between the 16-QAM and 8-QAM this margin increases to $2479 \mathrm{~km}(3311-832 \mathrm{~km})$. The larger margins contribute to increasing the number of connections that match the selected modulation format for either MF or MCF equivalent solutions. 

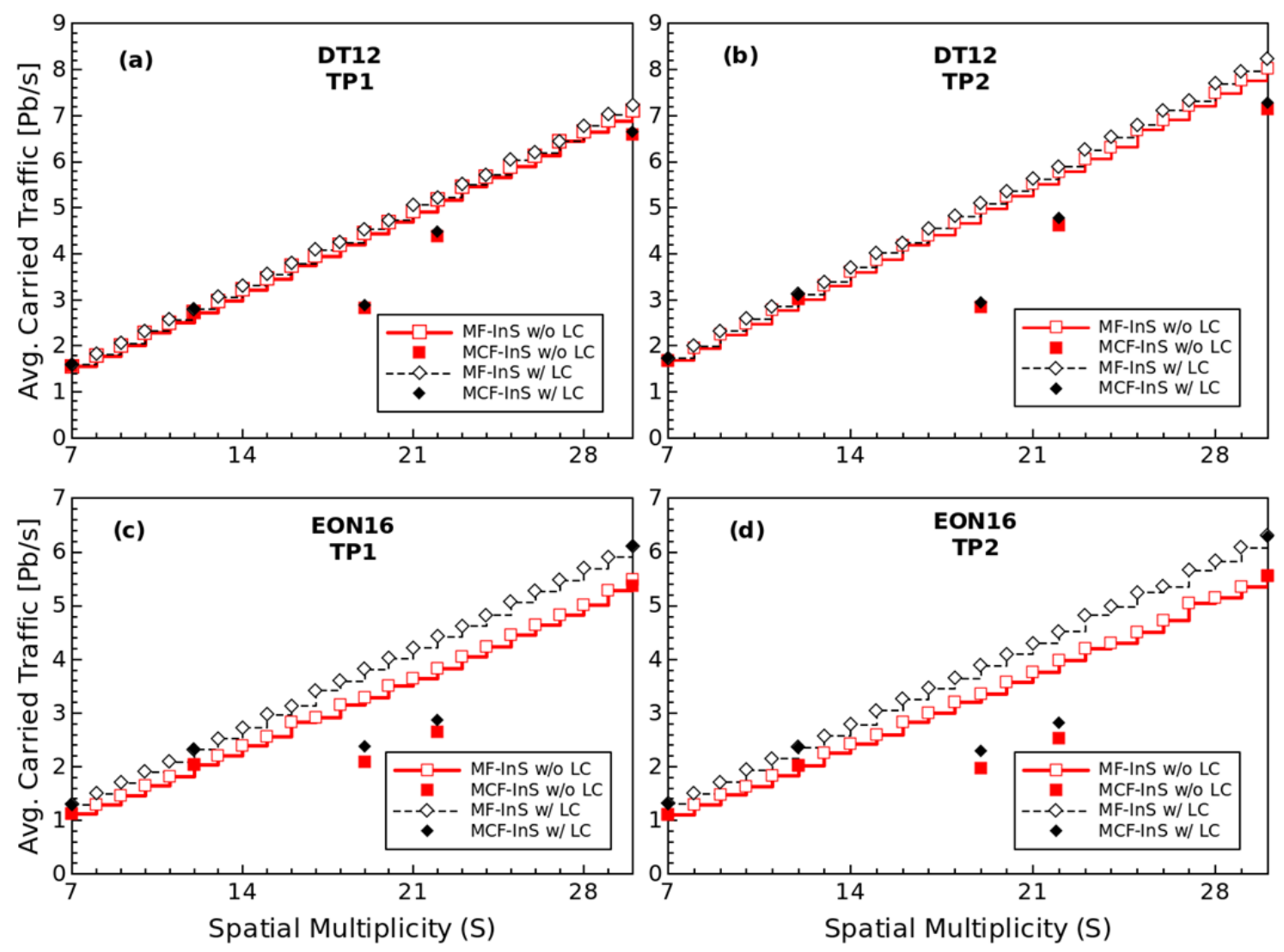

Fig. 4. Average Carried Traffic (in Pbps) vs. spatial multiplicity with Spe-SChs in: (a) DT12 network under TP1, (b) DT12 network under TP2, (c) EON16 network under TP1 and (d) EON16 network under TP2.

In turn, Fig. 5 shows the network throughput scalability of InS without LC support SDMROADM and of the benchmark SDM-ROADMs, namely, InS with LC support and JoS (with endto-end spatial traffic grooming) when supporting Spa-SChs. As observed, regardless the SDMROADM architecture, an $S$ increase does not always imply throughput increase. That is, the strictly increasing behavior of throughput versus spatial multiplicity for Spe-SCh allocation is not evidenced when Spa-SChs are allocated. This is caused by two factors: (i) the spectral grid constraint. For example, for $r_{d}=400 \mathrm{~Gb} / \mathrm{s}, S=7, \mathrm{~GB}=7.5 \mathrm{GHz}$ and Polarization Multiplexing (PM)-16QAM the resulting $n_{f s}$ is 2 , and when $S=8$ the $n_{f s}$ continues being equal to 2 ; and (ii) the spectral and spatial assignment of Spa-SCh has a greedy behavior, i.e., for a given demand if an $S$ increment yields lower spectral resources, this SCh configuration $\left(n_{f s}, n_{s}\right)$ is preferred. It may happen that sometimes the unused spatial resources (left by the greedy spatial assignment policy of Spa-SChs) for a given spectrum portion cannot be used by other lightpaths, because they do not suitably fit into them unless they are accordingly divided in several chunks. These two aspects, the spectral grid constraint and the unused spectral-spatial resources, cause the step-like shape in the three throughput curves when deploying InS $(\mathrm{w} /$ and $\mathrm{w} / \mathrm{O}$ LC) and JoS SDM-ROADM architectures (i.e., different from the strictly increasing behavior of the curves with Spe-SChs). We also observe that the performance of the InS without LC support SDM-ROADM architecture is practically equal (differences lower than $1 \%$ are reported) to the InS with LC support one in both MF and MCF scenarios. Finally, the before stated greedy behavior (in spectral-spatial resources assignment) of Spa-SCh as $S$ increases, causes not only that some points do not yield any throughput increment, but also a 
throughput drop. Hence, for Spa-SCh allocation, it seems that in some cases it may be convenient to explore MC-based Spa-SChs, i.e., Spa-SChs with higher $n_{f s}$ instead of preferring Spa-SChs with large Sb-Chs, although not considered in this work.

The results of Spa-SChs, as the ones of Spe-SChs, also evidence TP sensitivity. As demonstrated in [17], [24], [26], the performance of different SDM-ROADMs is influenced by the size and diversity of connections. Indeed, according to [26] when large connections are considered the performance of three SDM-ROADMs (InS without LC support, JoS, FJoS) are practically equal. The argument seems to be somewhat obvious, with large demands the unallocated spectralspatial resources are not able to be assigned to other demands under InS without LC support or FJoS schemes. Similar to results in [26], we have also found that, for low spatial channel count (see e.g., Fig. 5(d) and $S=12$ ) and large demands, the performance (in terms of blocking probability for [26] or in terms of throughput for this work) of two SDM-ROADMs (InS without LC support and JoS) is almost identical. As a contribution of this work, we show that the performance of InS with LC support (in spite of its flexibility) could also become identical to the one offered by the two other architectures. Nevertheless, according to our results, for higher spatial channel count (e.g., $S=18$ in Fig. 5(d)) -upgrade necessary e.g., due to the increment of the offered connections- this similarity in outcomes is not evidenced. In fact, the performance of InS (w/ or w/o LC) SDM-ROADMs in dynamic scenarios outperforms (up to 40\%) the one of JoS even enhancing its performance by applying end-to-end spatial traffic grooming, as result of flexibility provided by the former architectures. Then, the results from Fig. 4 and 5 reveal that the performance of SDM-ROADMs depends on both the traffic profile and the spatial multiplicity under Spe-Schs and Spa-SChs.

Specifically for MCF cases, as in Fig. 4, 19-core and 22-core MCFs report significant differences versus the MF equivalent solutions. With the 19-core MCF, i.e., the one with the highest ICXT, differences in terms of network throughput of up to $58 \%$ are observed in both networks, DT12 and EON16, compared to the equivalent MF solution. In the EON16 network, the throughput gain of the 19-core MCF versus the 12-core one is in the best cases up to $\sim 22 \%$ and $\sim 13 \%$ for TP1 and TP2, respectively, while for DT12 network is up to $\sim 5 \%$ and $\sim 2 \%$. That is, these results also evidence TP sensitivity. The higher the traffic demands, the higher the number of spatial channels that can be assigned to Spa-SChs, reducing the possibility that spatial and spectral resources are left unused (wasted). Consequently, the highest TP (i.e., the TP2 one) improves the network resources usage and evidences a $16 \%$ of network throughput increment regarding TP1 in EON16 for $S=30$, while for DT12 up to $\sim 42 \%$ network throughput increment is evidenced scaling the traffic profile from TP1 to TP2. In Fig. 5(d) is important to highlight the performance of different SDM-ROADM architectures for the 19- and 22-core MCFs. These MCFs report the highest ICXT impact and when they are required for long haul communications (in terms of physical distance) and large (in terms of bit-rate) connections under Spa-SChs, as observed, the ICXT dominates over the SDM-ROADM architectures. That is, whereas for MF-based solutions the performance of InS ( $w /$ and w/o LC support) SDMROADMs outperforms the JoS one, for MCF-based ones the performance of the three SDMROADMs are very similar. This happens because unused spectral-spatial resources per SCh cannot be assigned to other traffic demands, regardless of what switching strategy is considered. 

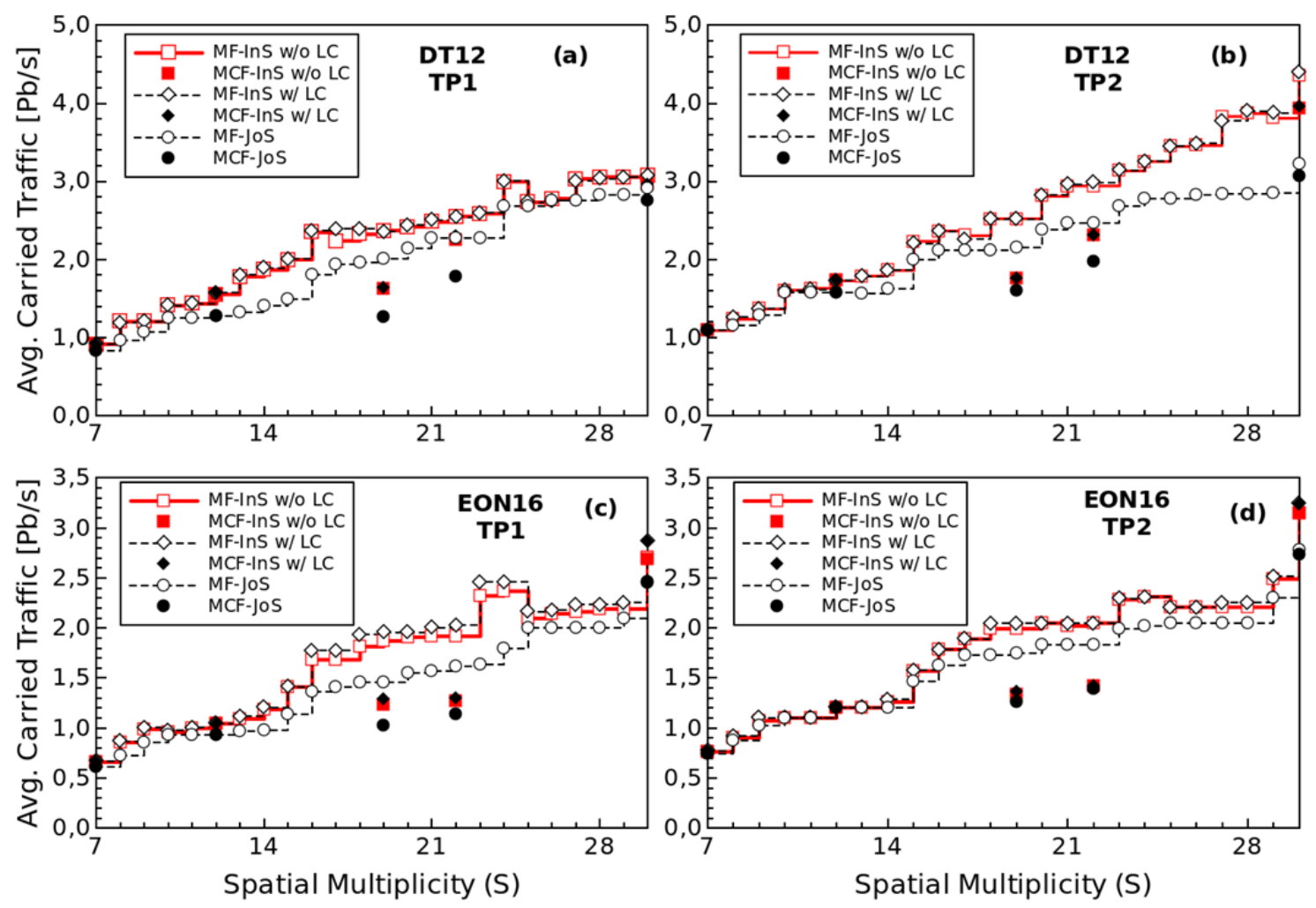

Fig. 5. Average Carried Traffic (in Pbps) vs. spatial multiplicity when supporting Spa-SChs in: (a) DT12 network under TP1, (b) DT12 network under TP2, (c) EON16 network under TP1 and (d) EON16 network under TP2.

One important aspect to analyse from the previous presented results is the network performance differences between the two versions of InS ( $w /$ and w/o LC support) SDMROADMs for MF versus MCF solutions. In Fig. 4(d), the largest performance differences between the two SDM-ROADM architectures are observed. However, these differences are very similar regardless of the SDM fibre (MCF or MF) for $S=19$ and 30 (up to $14 \%$ and $10 \%$, respectively), while for $S=22$ the impact of SCC is reduced by 2 percentage points ( $12 \%$ vs. $10 \%$ for MF- and MCF-based solutions, respectively). The most significant outcome from the latter aspect is that, ICXT does not contribute to the network performance degradation when SCC is considered, but can even be reduced depending on the ICXT value.

Another important analysis from our results is the comparison of the spatial multiplicity scalability with the throughput scalability under two different scenarios: (i) MF vs. MCF SDM solutions (ii) different SDM-ROADM architectures. The worst case of (i) -observed in Fig. 4(b)reports that a $4.3 \times(30 / 7)$ spatial multiplicity scalability entails a similar throughput scalability (7.8/1.8) for MF-based solution, while for MCF-based one the throughput scalability downs to $3.8 x(6.8 / 1.8)$. Likewise, the worst case of (ii) -observed in Fig. 5(b)- a 4.3x spatial multiplicity scalability yields a similar throughput scalability for InS ( $w /$ and $w / o$ LC), while for JoS (with end-to-end spatial traffic grooming) the throughput scalability downs to $3 x$.

Until now, we have analysed the impact of SCC in terms of throughput using the spatial multiplicity scalability criterion. Moreover, it is interesting to observe this impact in terms of BBP for increasing traffic (i.e., increasing the offered load). Fig. 6 shows the BBP vs. Offered Load (in Pbs) for some relevant scenarios. In particular, we consider for this analysis the InS (w/ and w/o LC support) SDM-ROADM architecture, EON16 network (where ICXT effects are 
more relevant), $S=12$ and $S=22$ (some intermediate spatial multiplicities where there exists a MCF prototype), traffic profile TP2 (which has shown throughput gains for the considered 7x to 30x spatial multiplicity range). Fig. 6(a,b) show the results for Spe-SCh, while Fig. 6(c,d) for SpaSCh. According to the results, when Spe-SChs and MF-based solutions are considered, the penalties (in terms of BBP) of InS w/o LC support architecture regarding InS w/ LC support one are more evident, ranging from 0.2 to 2 orders of magnitude for a BBP range from $10^{-1}$ to $10^{-5}$, respectively. For the cases of MCF-based solutions, as ICXT of the 12-core MCF has not impact on the TR, its performance is identical to $12-\mathrm{MF}$ equivalent solution, whereas for the 22-core MCF the penalties of SCC range from 0.2 to 1.3 orders of magnitude in terms of BBP. These results also show that, as stated before, the impact of SCC is reduced when 22-core MCFs are considered. Fig. 6(c,d) show the same analysis for Spa-SChs. In this case, as observed, the impact of SCC is practically non-existent (no impact of LC support). Finally, Fig. 6 clearly shows the differences in the offered load between Spe- and Spa-SChs. For example, for a maximum $10 \%$ BBP, networks allocating Spe-SChs can support up to $2 x$ more offered load than when they allocate Spa-SChs, due to spectrum savings of GBs.
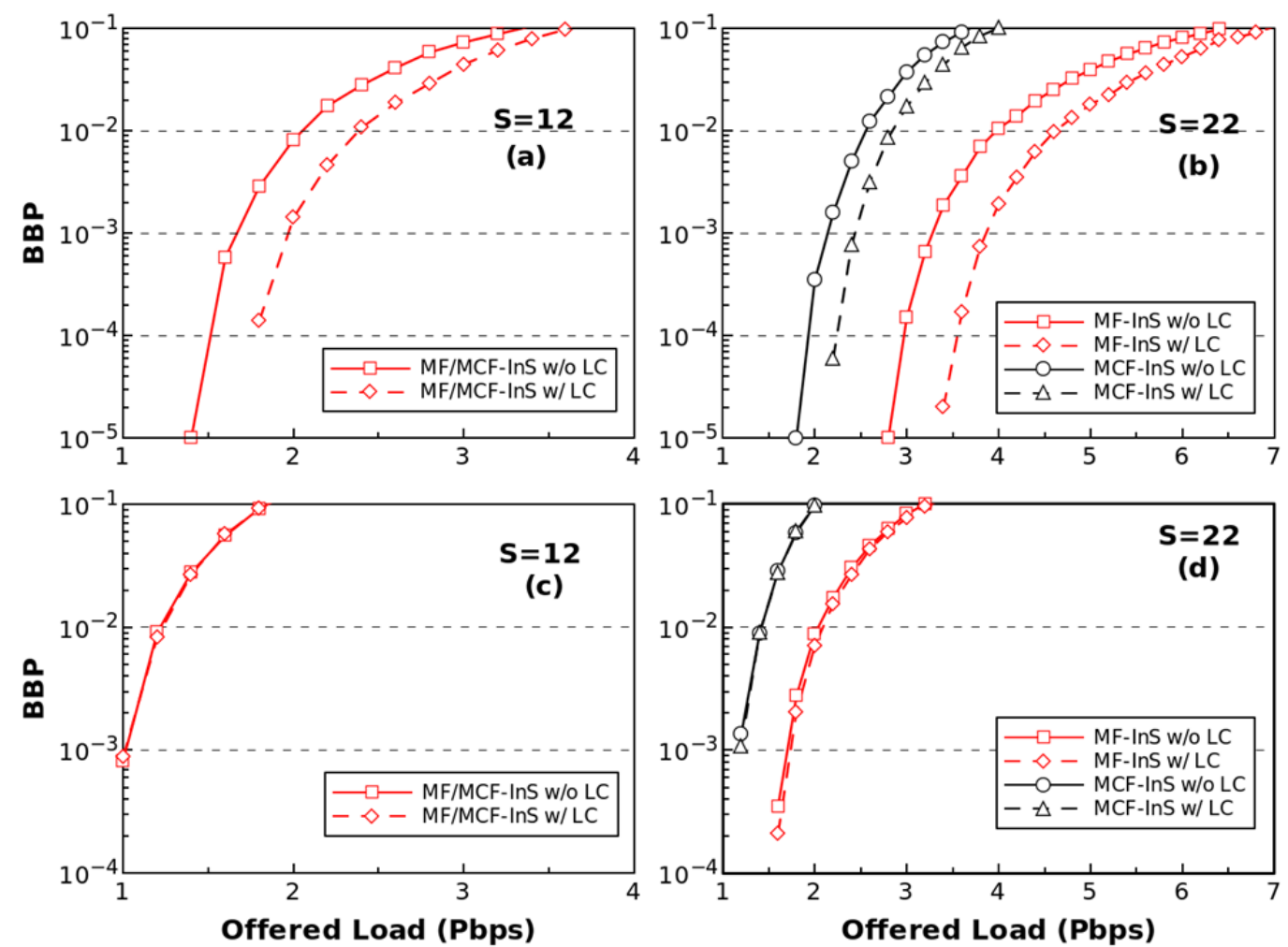

Fig. 6. BBP versus Offered Load (in Pbps) for EON16 network and TP2 when supporting: (a) SpeSChs, $S=12$, (b) Spe-SChs, $S=22$, (c) Spa-SChs, $S=12$ and (d) Spa-SChs, $S=22$.

To conclude this section, a cost analysis of the bypass part of different SDM-ROADM designs is carried out in order to give insight into the trade-off between network throughput and hardware cost. This cost analysis does not intend to be exhaustive because there are other important costs to be considered, e.g., the BVTs cost (the major network equipment cost in SDM networks [27]). To this end, the cost model presented in [16] regarding the SSSs and summarized in Table 4 is taken into account. The last row for $1 \times 320$ SSS has been extrapolated according to the port doubling and $58 \%$ premium cost criterion considered also in [16]. 
TABLE 4

Normalized Cost per SSS [16]

\begin{tabular}{|c|c|}
\hline SSS Size & $\begin{array}{c}\text { Normalized } \\
\text { Cost }\end{array}$ \\
\hline $1 \times 5$ & 0.63 \\
\hline $1 \times 9$ & 1 \\
\hline $1 \times 20$ & 1.58 \\
\hline $1 \times 40$ & 2.50 \\
\hline $1 \times 80$ & 3.95 \\
\hline $1 \times 160$ & 6.25 \\
\hline $1 \times 320$ & 9.87 \\
\hline
\end{tabular}

First, for each topology shown in Fig. 3, we quantify the number of SSSs per node and their required size, based on the previously presented Table 1 , in order to obtain the node-wide cost. Then, the total network-wide cost (i.e., the sum of all costs per node) is computed and its results are shown in Table 5 for 7/19/30 spatial channels (as examples cases) in the both reference topologies. This results show that the most expensive solution (considering the bypass part of the ROADMs) is the InS with LC support, followed by the InS without LC support and finally, the cheapest one appears to be the JoS SDM-ROADM solution. We determine that the InS with LC support SDM-ROADM solution is more and more expensive as the spatial channel count increases. Thus, for $S=7$ the InS with LC support solution is $2.9 \mathrm{x}$ and $2.5 \mathrm{x}$ more expensive than the InS without LC support one for DT12 and EON16 networks, respectively, while for $S=30$ these difference raise up to $7.1 \mathrm{x}$ and $6.3 \mathrm{x}$. Similarly, the InS without LC support SDM-ROADM solution is more and more expensive than the JoS one as $S$ increases. Cost increments up to $1.6 \mathrm{x}$ and $2 \mathrm{x}$ are reported for $S=7$ in DT12 and EON16 networks, respectively, while for $S=30$ these differences raise up to around $3 x$ for both networks.

In conclusion, the cost of the bypass part in InS w/o LC support SDM-ROADMs is reduced to a large extent compared to the InS w/ LC support solution, while the throughput capabilities remain very similar, with differences up to $14 \%$ when supporting Spe-SChs in large continental networks. Moreover, when supporting Spa-SChs, it is necessary to validate if the throughput gains of the InS w/o LC support SDM-ROADM versus the JoS one justify the extra cost of the network nodes. For instance in Fig. 5(b), when $S=30$ a throughput gain of $40 \%$ should be analysed in detail to determine if the $3 x$ cost network-wide increment of the InS w/o LC support SDM-ROADM solution is justified; otherwise, for these scenarios the JoS solution could become the best option. It is worth mentioning that, Spa-SChs against Spe-SChs can reduce the cost of the BVTs, resulting from the absence of frequency combs or AWGs, the use of common laser sources and joint digital signal processing at the receivers, as we mentioned in Section 3. According to [27], this reduction can be around 5-20\% for Spa-SChs with 2-10 Sb-Chs.

TABLE 5

Network-wide cost

\begin{tabular}{|c|c|c|}
\hline \multirow{2}{*}{ SDM-ROADM } & \multicolumn{2}{|c|}{$\begin{array}{r}\text { Network-wide cost } \\
\text { (arbitrary units) } \\
\text { for } S=7 / 19 / 30\end{array}$} \\
\cline { 2 - 3 } & DT12 & EON16 \\
\hline InS w/o LC support & $106 / 287 / 454$ & $141 / 383 / 604$ \\
\hline InS w/ LC support & $301 / 1325 / 3222$ & $352 / 1511 / 3771$ \\
\hline JoS & $66 / 113 / 165$ & $71 / 140 / 200$ \\
\hline
\end{tabular}




\section{Conclusions and Future Work}

We have assessed the InS without LC support SDM-ROADM architecture in online scenarios against InS with LC support and JoS SDM-ROADM benchmark solutions, when supporting either Spe-SChs or Spa-SChs. In terms of network throughput, InS without LC support versus InS with LC support solutions can lead to a reduction between 0\% (the minimum obtained when Spa-SChs are considered) and 14\% (the maximum obtained when Spe-SChs are considered). Moreover, ICXT does not contribute to performance degradation when SCC is considered. It is worth highlighting that these results are in the order of the ones obtained in our previous work $[11,15]$ for offline scenarios. In contrast, the InS without LC support SDMROADM solution allows carrying up to $40 \%$ extra network throughput than the JoS SDMROADM solution. We have found that the performance (as stated in [26]) of the SDM-ROAMDs is not only influenced by the traffic profile, but also by the spatial multiplicity, thus extending its dependency on both parameters under Spe-SChs and Spa-SChs. In any case, there exists a trade-off between network throughput gains and network nodes complexity. The InS without LC support SDM-ROADM becomes a cheaper solution than the InS with LC support one, reducing the cost of SDM-ROADMs (bypass part) down to $86 \%$ with minimum throughput penalties. In contrast, it can be up to $3 x$ more expensive than the JoS one. We have found that the CAPEX of SDM-ROADMs is also influenced by the spatial multiplicity. For instance, the CAPEX of InS with LC support is from 2.5x to $7.1 x$ higher than the one of InS without LC support for a $4.3 x$ spatial multiplicity increase.

In addition, the scalability of the spatial multiplicity in some cases is not strictly equivalent to scalability produced over the throughput. Thus, for a $4.3 \mathrm{x}$ spatial multiplicity (studied in this work) yields a 3.8x for MCF solutions and 2.9x for JoS SDM-ROADM (worst cases). Regarding MCF solutions, the 19- and 22-core MCFs considered in this work evidence the highest throughput degradation versus the MF equivalent solutions, becoming up to $58 \%$ and $70 \%$ when allocating Spa-SCh and Spe-SCh, respectively.

Finally, a combination of Spa-SChs (SC or MC) and Spe-SChs can be explored to evaluate their impact in the overall network performance, which is intended to be addressed in a future work. Additionally, a more complete cost analysis of SDM networks including more network elements, like add/drop ROADM part, amplifiers, BVTs, etc., can be also considered as a future work.

\section{Acknowledgements}

This work has been partially funded by the Spanish national projects SUNSET (TEC2014-59583C2-1-R), which receives funding from FEDER, Elastic Networks (TEC2015-71932-REDT), ONOFRE (TEC2014-53071-C3-1-P) and (ONOFRE-2 TEC2017-84423-C3-2-P) projects.

Rubén Rumipamba is recipient of a full scholarship from Secretaría Nacional de Ciencia y Tecnología (SENESCYT) - Ecuador.

\section{References}

[1] O. Gerstel, M. Jinno, A. Lord, and S. J. Ben Yoo, "Elastic optical networking: A new dawn for the optical layer?," IEEE Commun. Mag., vol. 50, no. 2, pp. 12-20, 2012. 
[2] P. J. Winzer, "Making spatial multiplexing a reality," Nat. Photonics, vol. 8, no. 5, pp. 345-348, Apr. 2014.

[3] International Telecommunication Union - ITU-T, "G.694.1 (02/2012), Spectral grids for WDM applications: DWDM frequency grid," Ser. G.694.1, pp. 1-16, 2012.

[4] P. J. Winzer, "Spatial Multiplexing : The Next Frontier in Network Capacity Scaling," Eur. Conf. Exhib. Opt. Commun., p. We.1.D.1, 2013.

[5] D. J. Richardson, J. M. Fini, and L. E. Nelson, "Space Division Multiplexing in Optical Fibres," Nat. Photonics, vol. 7, pp. 354-362, 2013.

[6] J. Sakaguchi, Y. Awaji, N. Wada, A. Kanno, T. Kawanishi, T. Hayashi, T. Taru, T. Kobayashi, and M. Watanabe, "Space division multiplexed transmission of 109-Tb/s data signals using homogeneous seven-core fiber," J. Light. Technol., vol. 30, no. 4, pp. 658-665, 2012.

[7] A. Sano, H. Takara, T. Kobayashi, H. Kawakami, H. Kishikawa, T. Nakagawa, Y. Miyamoto, Y. Abe, H. Ono, K. Shikama, M. Nagatani, T. Mori, Y. Sasaki, I. Ishida, K. Takenaga, S. Matsuo, K. Saitoh, M. Koshiba, M. Yamada, H. Masuda, and T. Morioka, "409-Tb/s + 409-Tb/s crosstalk suppressed bidirectional MCF transmission over $450 \mathrm{~km}$ using propagation-direction interleaving.," Opt. Express, vol. 21, no. 14, pp. 1677716783, 2013.

[8] J. Sakaguchi, W. Klaus, B. J. Puttnam, J. M. D. Mendinueta, Y. Awaji, N. Wada, Y. Tsuchida, K. Maeda, M. Tadakuma, K. Imamura, R. Sugizaki, T. Kobayashi, Y. Tottori, M. Watanabe, and R. V Jensen, "19-core MCF transmission system using EDFA with shared core pumping coupled via free-space optics.," Opt. Express, vol. 22, no. 1, pp. 90-5, 2014.

[9] B. J. Puttnam, R. S. Luis, W. Klaus, J. Sakaguchi, J.-M. Delgado Mendinueta, Y. Awaji, N. Wada, Y. Tamura, T. Hayashi, M. Hirano, and J. Marciante, "2.15 Pb/s transmission using a 22 core homogeneous single-mode multi-core fiber and wideband optical comb," in 2015 European Conference on Optical Communication (ECOC), 2015, pp. 1-3.

[10] Y. Amma, Y. Sasaki, K. Takenaga, S. Matsuo, J. Tu, K. Saitoh, M. Koshiba, T. Morioka, and Y. Miyamoto, "High-density Multicore Fiber with Heterogeneous Core Arrangement," in Optical Fiber Communication Conference, 2015, p. Th4C.4.

[11] R. Rumipamba-Zambrano, F.-J. Moreno-Muro, P. Pavon-Marino, J. Perello, S. Spadaro, and J. Sole-Pareta, "Assessment of Flex-Grid/MCF Optical Networks with ROADM limited core switching capability," in 2017 21st International Conference on Optical Network Design and Modeling, ONDM 2017 - Conference Proceedings, 2017.

[12] D. M. Marom and M. Blau, "Switching solutions for WDM-SDM optical networks," IEEE Commun. Mag., vol. 53, no. 2, pp. 60-68, 2015.

[13] N. Amaya, M. Irfan, G. Zervas, R. Nejabati, D. Simeonidou, J. Sakaguchi, W. Klaus, B. J. Puttnam, T. Miyazawa, Y. Awaji, N. Wada, and I. Henning, "Fully-elastic multi-granular network with space/frequency/time switching using multi-core fibres and programmable optical nodes," Opt. Express, vol. 21, no. 7, p. 8865, Apr. 2013.

[14] D. M. Marom, P. D. Colbourne, A. D'Errico, N. K. Fontaine, Y. Ikuma, R. Proietti, L. Zong, J. M. Rivas-Moscoso, and I. Tomkos, "Survey of Photonic Switching Architectures and Technologies in Support of Spatially and Spectrally Flexible Optical Networking [Invited]," J. Opt. Commun. Netw., vol. 9, no. 1, p. 1, Jan. 2017.

[15] F.-J. Moreno-Muro, R. Rumipamba-Zambrano, P. Pavón-Marino, J. Perelló, J. M. Gené, and S. Spadaro, "Evaluation of Core-Continuity-Constrained ROADMs for Flex-Grid/MCF Optical Networks," J. Opt. Commun. Netw., vol. 9, no. 11, p. 1041, Nov. 2017.

[16] J. M. Rivas, B. Shariati, D. M. Marom, J. M. Rivas-Moscoso, B. Shariati, D. M. Marom, D. Klonidis, I. Tomkos, and I. Tomkos, "Comparison of CD ( C ) ROADM Architectures for 
Space Division Multiplexed Networks," Opt. Fiber Commun. Conf., no. C, pp. 3-5, Mar. 2017.

[17] B. Shariati, J. M. Rivas-Moscoso, D. M. Marom, S. Ben-Ezra, D. Klonidis, L. Velasco, and I. Tomkos, "Impact of spatial and spectral granularity on the performance of SDM networks based on spatial superchannel switching," J. Light. Technol., vol. 35, no. 13, pp. 2559-2568, 2017.

[18] J. Perelló, J. M. Gené, A. Pagès, J. A. Lazaro, and S. Spadaro, "Flex-Grid/SDM Backbone Network Design with Inter-Core XT-Limited Transmission Reach," J. Opt. Commun. Netw., vol. 8, no. 8, p. 540, Aug. 2016.

[19] H. Tode and Y. Hirota, "Routing, spectrum and core assignment for space division multiplexing elastic optical networks," 2014 16th Int. Telecommun. Netw. Strateg. Plan. Symp., 2014.

[20] A. Muhammad, G. Zervas, and R. Forchheimer, "Resource Allocation for Space-Division Multiplexing: Optical White Box Versus Optical Black Box Networking," J. Light. Technol., vol. 33, no. 23, Dec. 2015.

[21] L. E. Nelson, M. D. Feuer, K. Abedin, X. Zhou, T. F. Taunay, J. M. Fini, B. Zhu, R. Isaac, R. Harel, G. Cohen, and D. M. Marom, "Spatial superchannel routing in a two-span ROADM system for space division multiplexing," J. Light. Technol., vol. 32, no. 4, pp. 783-789, 2014.

[22] D. Klonidis, F. Cugini, O. Gerstel, M. Jinno, V. Lopez, E. Palkopoulou, M. Sekiya, D. Siracusa, G. Thouénon, and C. Betoule, "Spectrally and spatially flexible optical network planning and operations," IEEE Commun. Mag., vol. 53, no. 2, pp. 69-78, 2015.

[23] P. Sayyad Khodashenas, J.-M. Rivas-Moscoso, D. Siracusa, F. Pederzolli, B. Shariati, D. Klonidis, E. Salvadori, and I. Tomkos, "Comparison of Spectral and Spatial Super-channel Allocation Schemes for SDM Networks," J. Light. Technol., vol. 8724, no. c, pp. 1-1, 2016.

[24] F. Pederzolli, D. Siracusa, B. Shariati, J. M. Rivas-Moscoso, E. Salvadori, and I. Tomkos, "Improving Performance of Spatially Joint-Switched Space Division Multiplexing Optical Networks via Spatial Group Sharing," J. Opt. Commun. Netw., vol. 9, no. 3, p. B1, Mar. 2017.

[25] B. Shariati, P. Sayyad Khodashenas, J. M. Rivas Moscoso, S. Ben-Ezra, D. Klonidis, F. Jimenez, L. Velasco, I. Tomkos, P. S. Khodashenas, J. M. Rivas-moscoso, and S. Ben-Ezra, "Evaluation of the Impact of Different SDM Switching Strategies in a Network Planning Scenario," Opt. Fiber Commun. Conf., no. 1, pp. 6-8, 2016.

[26] B. Shariati, D. Klonidis, D. Siracusa, F. Pederzolli, and L. Velasco, "Impact of Traffic Profile on the Performance of Spatial Superchannel Switching in SDM Networks," 42nd Eur. Conf. Exhib. Opt. Commun., no. M.1.F.1, pp. 73-75, 2016.

[27] J. M. Rivas-Moscoso, B. Shariati, A. Mastropaolo, D. Klonidis and I. Tomkos, "Cost Benefit Quantification of SDM Network Implementations based on Spatially Integrated Network Elements," 42nd Eur. Conf. Exhib. Opt. Commun., pp. 1-3, 2016.

[28] A. Muhammad, G. Zervas, D. Simeonidou, and R. Forchheimer, "Routing, Spectrum and Core Allocation in Flexgrid SDM Networks with Multi-core Fibers," Ondm. pp. 19-22, 2014.

[29] S. Fujii, Y. Hirota, H. Tode, and K. Murakami, "On-demand spectrum and core allocation for reducing crosstalk in multicore fibers in elastic optical networks," IEEE/OSA J. Opt. Commun. Netw., vol. 6, no. 12, Dec. 2014.

[30] T. Mizuno, H. Takara, K. Shibahara, A. Sano, and Y. Miyamoto, "Dense space division multiplexed transmission over multicore and multimode fiber for long-haul transport systems," J. Light. Technol., vol. 34, no. 6, pp. 1484-1493, 2016. 
[31] M. Ruiz, L. Velasco, A. Lord, D. Fonseca, M. Pióro, R. Wessäly, and J. P. FernándezPalacios, "Planning fixed to flexgrid gradual migration: Drivers and open issues," IEEE Commun. Mag., vol. 52, no. 1, pp. 70-76, 2014.

[32] M. M. Filer and S. Tibuleac, "N-degree ROADM Architecture Comparison: Broadcastand-Select versus Route-and-Select in $120 \mathrm{~Gb} / \mathrm{s}$ DP-QPSK Transmission Systems," in Optical Fiber Communication Conference, 2014, p. Th11.2.

[33] B. Collings, "New devices enabling software-defined optical networks," IEEE Commun. Mag., vol. 51, no. 3, pp. 66-71, Mar. 2013.

[34] "Lumentum: New ROADM Whitebox Preliminary Datasheet" [Online]. Available: http://www.bktel.com/index_htm_files/ROADM\%20Whitebox\%20Target\%20Specs.pdf [Last accessed: Jan. 2018].

[35] E. Palkopoulou, G. Bosco, A. Carena, D. Klonidis, P. Poggiolini, and I. Tomkos, "NyquistWDM-Based Flexible Optical Networks: Exploring Physical Layer Design Parameters," J. Light. Technol., vol. 31, no. 14, pp. 2332-2339, Jul. 2013.

[36] R. Rumipamba-Zambrano, J. Perelló, J. M. J. M. Gené, and S. Spadaro, "Cost-effective spatial super-channel allocation in Flex-Grid/MCF optical core networks," Opt. Switch. Netw., vol. 27, pp. 93-101, Jan. 2018.

[37] R. Rumipamba-Zambrano, J. Perelló, J. M. Gené, and S. Spadaro, "Capacity quantification of joint-switching-enabled Flex- Grid/SDM optical backbone networks," in Optics InfoBase Conference Papers, 2017, vol. Part F40-O.

[38] J. M. Rivas-Moscoso, S. Ben-Ezra, P. S. Khodashenas, D. M. Marom, D. Klonidis, P. Zakynthinos, and I. Tomkos, "Cost and power consumption model for flexible superchannel transmission with all-optical sub-channel add/drop capability," Int. Conf. Transparent Opt. Networks, vol. 2015-Augus, pp. 1-4, 2015.

[39] M. D. Feuer, L. E. Nelson, X. Zhou, S. L. Woodward, R. Isaac, Benyuan Zhu, T. F. Taunay, M. Fishteyn, J. M. Fini, and M. F. Yan, "Joint Digital Signal Processing Receivers for Spatial Superchannels," IEEE Photonics Technol. Lett., vol. 24, no. 21, pp. 1957-1960, Nov. 2012. 